\title{
Lentivector-mediated $S M N$ replacement in a mouse model of spinal muscular atrophy
}

\author{
Mimoun Azzouz,, ${ }^{1}$ Thanh Le, ${ }^{2}$ G. Scott Ralph,, ${ }^{1}$ Lucy Walmsley, ${ }^{1}$ Umrao R. Monani, ${ }^{2}$ \\ Debbie C.P. Lee, ${ }^{1}$ Fraser Wilkes, ${ }^{1}$ Kyriacos A. Mitrophanous, ${ }^{1}$ Susan M. Kingsman, ${ }^{1}$ \\ Arthur H.M. Burghes, ${ }^{2}$ and Nicholas D. Mazarakis ${ }^{1}$
}

10xford BioMedica plc, Oxford, United Kingdom. ${ }^{2}$ Department of Molecular and Cellular Biochemistry, Molecular Genetics and Neurology, Ohio State University, Columbus, Ohio, USA.

\begin{abstract}
Spinal muscular atrophy (SMA) is a frequent recessive autosomal disorder. It is caused by mutations or deletion of the telomeric copy of the survival motor neuron $(S M N)$ gene, leading to depletion in SMN protein levels. The treatment rationale for SMA is to halt or delay the degeneration of motor neurons, but to date there are no effective drug treatments for this disease. We have previously demonstrated that pseudotyping of the nonprimate equine infectious anemia virus (using the lentivector gene transfer system) with the glycoprotein of the Evelyn-Rokitnicki-Abelseth strain of the rabies virus confers retrograde axonal transport on these vectors. Here, we report that lentivector expressing human $S M N$ was successfully used to restore SMN protein levels in SMA type 1 fibroblasts. Multiple single injections of a lentiviral vector expressing $S M N$ in various muscles of SMA mice restored SMN to motor neurons, reduced motor neuron death, and increased the life expectancy by an average of 3 and 5 days $(20 \%$ and $38 \%)$ compared with LacZ and untreated animals, respectively. Further extension of survival by SMN expression constructs will likely require a knowledge of when and/or where high levels of SMN are needed.
\end{abstract}

\section{Introduction}

Spinal muscular atrophy (SMA) is one of the most common genetic diseases leading to death in childhood. SMA is characterized by muscle weakness caused by degeneration of motor neurons (MNs) in the spinal cord and brain stem nuclei $(1,2)$. It is caused by mutations or deletion of the telomeric copy (SMN1) of the survival motor neuron (SMN) gene, leading to depletion in SMN protein levels (3). Based on the clinical severity and age at onset, the childhood SMAs have been divided into 3 types $(4,5)$. Type I SMA is the most severe, with onset occurring by 6 months of age and death by 2 years of age. The human SMN gene is located within a duplicated region, and both SMN genes are expressed. The 2 genes differ essentially by a single nucleotide that affects an exon splice enhancer, resulting in the majority of the transcript from SMN2 lacking exon $7(6,7)$. SMN2 does produce some full-length transcript and therefore stable SMN protein, but the levels are reduced; for an unknown reason this has a particular adverse effect on MNs. In contrast to humans, mice have a single gene $(S m n)$ that is equivalent to SMN1. Homozygous loss of this gene is lethal to embryos and results in massive cell death (8), which indicates that the SMN gene product is necessary for cellular survival and function. The introduction of 2 copies of $S M N 2$ into mice lacking $S m n$ rescues the embryonic lethality, resulting in mice with the SMA phenotype (9). A high copy number of SMN2 rescues the mice because sufficient SMN protein is

Nonstandard abbreviations used: ALS, amyotrophic lateral sclerosis; CGRP, calcitonin gene-related peptide; ChAT, choline acetyltransferase; EIAV, equine infectious anemia virus; gems, gemini of coiled bodies; MN, motor neuron; SMA, spinal muscular atrophy; SMN, survival motor neuron.

Conflict of interest: M. Azzouz, G.S. Ralph, L. Walmsley, D.C.P. Lee, F. Wilkes, K.A. Mitrophanous, S.M. Kingsman, and N.D. Mazarakis hold shares at Oxford BioMedica.

Citation for this article: J. Clin. Invest. 114:1726-1731 (2004).

doi:10.1172/JCI200422922. produced in MNs. The question becomes how to deliver SMN to sufficient MNs. We recently developed a novel lentiviral gene transfer system (lentivector) to deliver transgenes to neurons $(10-12)$. Equine infectious anemia virus (EIAV) vectors provide stable, long-term expression of exogenous genes after integration into the DNA of the host cell. For example, we recently reported that this particular viral vector led to long-term efficacy and correction of the amyotrophic lateral sclerosis (ALS) mouse model for over 5 months (12). The marker $L a c Z$ gene was expressed in the rat striatum for more than 5 months after transduction (10). Here, we have generated lentivector expressing human SMN and successfully used it to restore gemini of coiled bodies (gems) in type I SMA fibroblasts. Lentivector-mediated SMN gene therapy in SMA mice improved MN survival and induced a small but still significant increase in survival.

\section{Results}

Lentivector-mediated gene transfer in neonate mice. We first investigated the ability of rabies $\mathrm{G}$ pseudotyped EIAV-based lentivector to transduce $\mathrm{MNs}$ in the spinal cord and brain stem following viral injections into muscles of P2 FVB WT mice. P2 was chosen because this day was prior to the point of MN cell body loss in the SMA mice (13). One week after injection of high-titer lentivectorLacZ (approximately $10^{9}$ transducing units [T.U.]/ml) unilaterally in the gastrocnemius and facial muscle, strong reporter gene expression was observed in both the spinal cord (Figure 1, A and E), brain stem (Figure 1, B, C, and E), and muscle (Figure 1D). When staining for calcitonin gene-related peptide (CGRP) to identify the MNs more directly, we found that all $\beta$-gal-labeled neurons were also stained for CGRP (Figure 1, F and G). CGRP$\beta$-gal double labeling of spinal cord sections revealed that more than $70 \%$ of CGRP-positive MNs were transduced. Gene transfer was specific for MNs because LacZ was not detectable in other cell types (i.e., glial cells, vascular cells). Retrograde transport of 

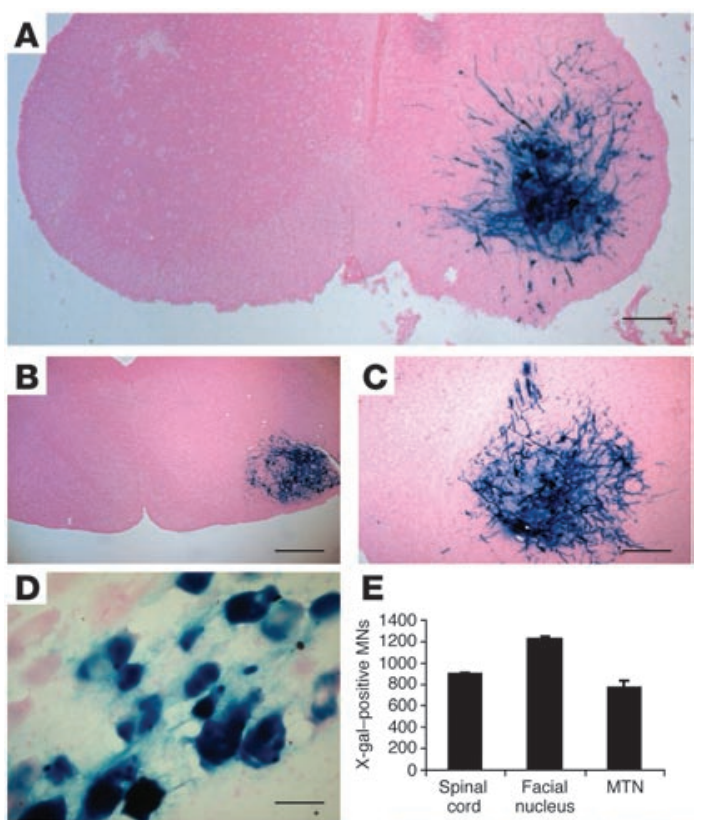

E
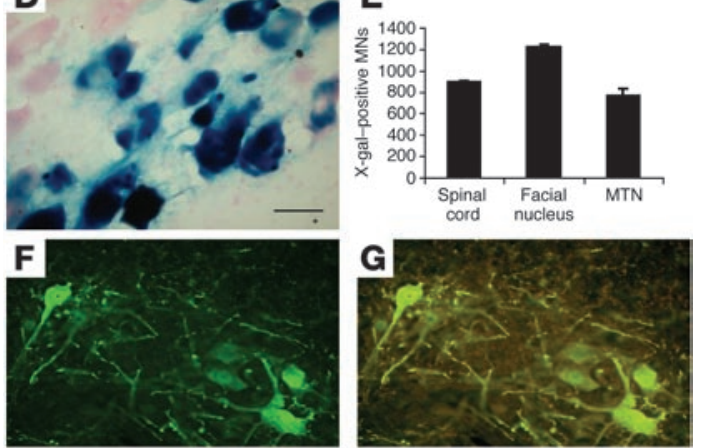

viral vector itself was confirmed by quantitative PCR (Taqman) experiments using sections taken from facial nucleus area, revealing that viral DNA could only be amplified from this area after rabies-G pseudotyped EIAV facial muscle transduction (data not shown). These findings further indicate that the EIAV-LacZ vectors were indeed retrogradely transported from the ipsilaterally injected muscle to the MNs in the spinal cord where the LacZ transgene was expressed unilaterally.

Lentivector-SMN restores SMN protein in SMA type 1 fibroblasts. The SMN protein is a $38-\mathrm{kDa}$, ubiquitously expressed protein found in the cytoplasm and nucleus $(14,15)$. In the nucleus of most cells SMN is concentrated in structures termed gems, which often colocalize with coiled bodies (16). In tissues and cells from type I SMA patients SMN levels are severely reduced and cells have few or no gems $(15,17)$. We have constructed lentivector (EIAV) expressing SMN or epitope-tagged HA-SMN (Figure 2A). Prior to in vivo gene transfer in SMA mice, we tested the ability of these constructs to express SMN in a type I SMA fibroblast cell line (Figure 2). We used fibroblasts from type I SMA patients because these cells produce very few or no gems (18). High SMN protein levels were detected in fibroblasts that had been incubated with lentivector-SMN, that is, up to 7 gems per cell were obtained in fibroblasts transduced with lentivector-SMN (Figure 2B). Gem counts revealed that the number of gems in lentivectorSMN-transduced cells was approximately twice that observed in normal fibroblasts (Figure 2J). Very few or no gems were detected in LacZ-transduced SMA patient-derived fibroblasts (Figure 2, C and J). To demonstrate that these structures are indeed gems, we performed double labeling of SMN and other proteins normally associated with nuclear gems, such as gemin2 (Figure 2, D-F). Western blots of transduced fibroblasts also revealed high SMN

\section{Figure 1}

EIAV-based lentivector-mediated gene transfer in vivo. Transverse sections of lumbar spinal cord (A), brain stem (B and $\mathbf{C}$ ), and muscle (D) showing transduction of both muscle and MNs after injection of $30 \mu \mathrm{l}$ rabies $\mathrm{G}$ pseudotyped lentivector-LacZ vector in the gastrocnemius $(\mathbf{A})$ and facial muscles (B and $\mathbf{C}$ ) of postnatal P2 FVB mice. (E) Cell counts of total transduced MNs in the lumbar spinal cord, facial nucleus, and motor trigeminal nucleus (MTN) after intramuscular injections of lentivector-LacZ into 2-day-old SMA mice. Data are means \pm SEM. Expression of $\beta$-gal (green) (F) colocalizes with the immunofluorescence of CGRP (red) in spinal MNs, producing yellow staining (G). Scale bars: $400 \mu \mathrm{m}$ (A and B), $200 \mu \mathrm{m}$ (C and D), $100 \mu \mathrm{m}$ (F and $\mathbf{G}$ ). levels when the SMN construct was used (Figure $2 \mathrm{~K}$ ) compared with LacZ and mock cells. Taken together, these data indicate high efficiency of lentivector expressing SMN to restore gems in SMA patient-derived fibroblasts.

Lentivector-mediated SMN replacement in SMA mice. The SMA mice used in the present study were generated as reported (13). These mice survive for a mean of $13.27 \pm 0.34$ days and showed significant weight differences by 5 days of age. The SMA mouse model displays $\mathrm{MN}$ cell body loss at 9 days of age. To explore the efficacy of SMN gene transfer on MN survival in the SMA mouse model, lentivector-SMN or lentivector-LacZ vectors were injected bilaterally into the hind limb gastrocnemius, diaphragm, intercostal, facial, and tongue muscles of SMA mice at disease onset at 2 days of age. Each muscle received single injections into multiple sites. We intentionally chose these sites of injection, because these muscles are critical for mobility (hind limb), respiration (diaphragm and intercostal muscles), and feeding (facial, tongue) and thus together determine general health and survival of the mice. A total of 5 SMA animals were injected with SMN-expressing lentivector and 5 with LacZexpressing vector. Five untreated animals were also included in the study. Analysis of their body weight revealed significant weight loss of LacZ-injected SMA mice starting between 4 and 5 days of age (Figure 3A). The introduction of the SMN construct delayed the weight loss by 2-3 days, however, compared with LacZ-injected animals (Figure 3A). The behavior of the animals followed the weight profile with treated animals showing delayed phenotypic presentation and losing the ability to ambulate slightly later.

The mice were killed at the clinical end point when they had difficulty feeding, had a clear downward trend, and demonstrated 


\section{A}
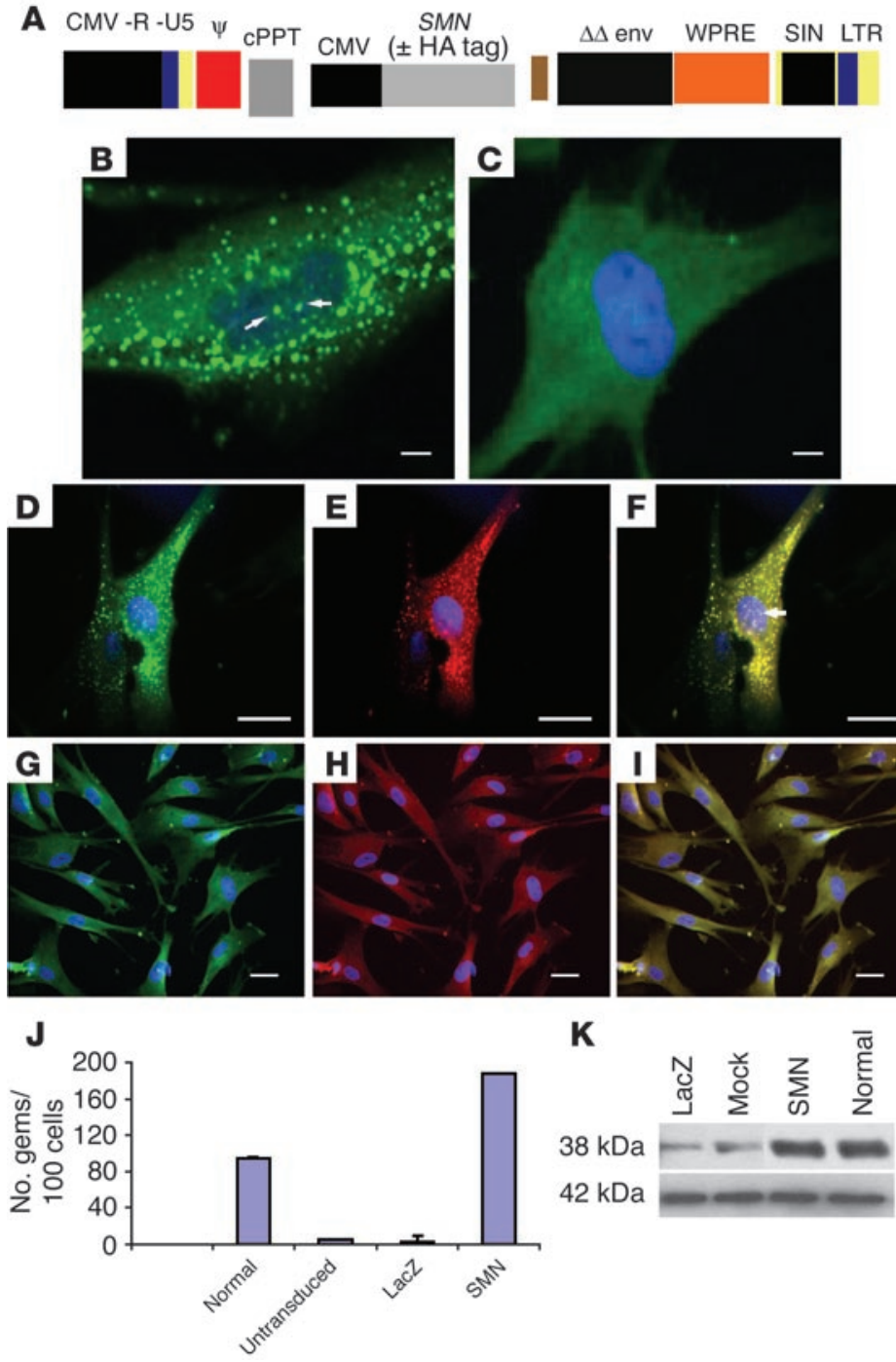

$\mathbf{K}$
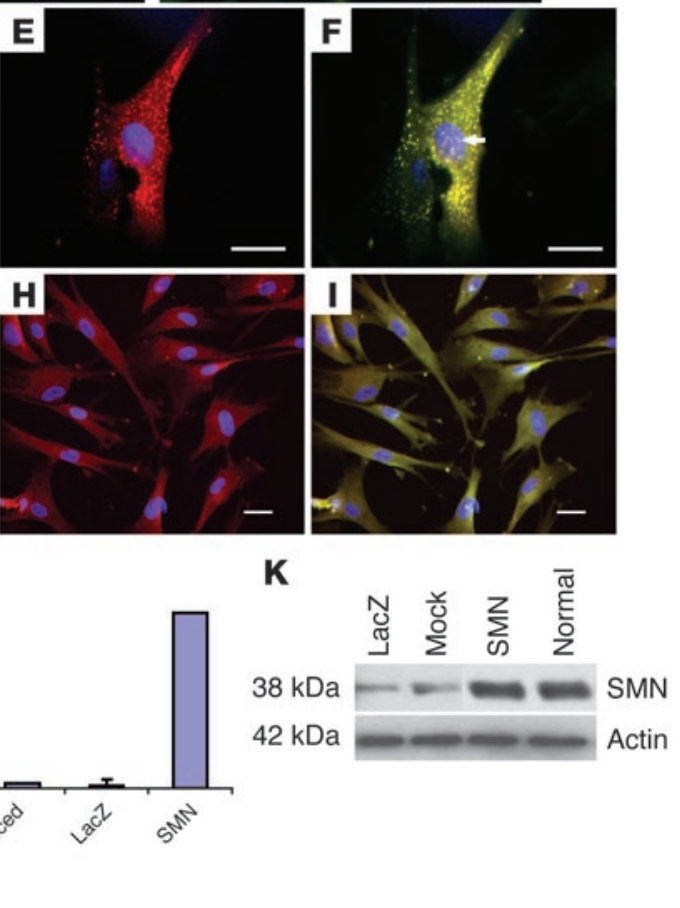

Figure 2

Lentivector-mediated restoration of SMN protein expression in vitro. (A) Schematic representation of Lentivector encoding for human SMN gene. CMV, cytomegalovirus; cPPT, central polypurine tract; $\Delta \Delta$ env, double-deleted envelope leaving only rev response element; WPRE, Woodchuck hepatitis virus posttranscriptional regulation element; SIN, self-inactivating; LTR, long-terminal repeat. (B) Lentivector-mediated expression of SMN in fibroblasts from type I SMA patients. Lentivector-SMN restores SMN expression in gems (arrows). No restoration of gems was observed in fibroblasts incubated with lentivector-LacZ (C). Expression of SMN in human fibroblasts (green) (D) colocalizes with the red immunofluorescence of gemin2 (E), producing yellow staining $(\mathbf{F})$. Arrow indicates colocalization of SMN with gemin2 in gems. Lentivector-LacZ-treated fibroblasts stained with SMN Abs (G) and gemin2 (H). (I) Merged image from $\mathbf{G}$ and $\mathbf{H}$. (J) Gem counts in lentivector-SMN-treated and control fibroblast cells. (K) Immunoblot confirming lentivector-mediated SMN expression in human fibroblasts using Abs against SMN. Scale bars: $50 \mu \mathrm{m}$ (B and C), $100 \mu \mathrm{m}$ (D-F), $200 \mu \mathrm{m}(\mathbf{G}-\mathbf{I})$.
To monitor transgene expression in vivo, we used a lentivector encoding a HA epitope-tagged version of SMN. HA labeling at the end stage of disease revealed that lentivector-derived SMN protein was expressed in approximately $450 \pm 15 \mathrm{MNs}$ in the ventral horn of lumbar spinal cord (Figure 3, C and D). No HA staining was observed in LacZ-injected mice (Figure 3E). To further demonstrate gene transfer with lentivector-SMN vectors, Western blots of ventral horn spinal cord from SMN-treated mice revealed high SMN levels compared with LacZ-injected animals (Figure $3 \mathrm{H})$. Taken together, the above results are indicative that gene transfer using lentivector expressing SMN at onset of disease induces not only an extension in life span, but also results in a delay in the motor phenotype in a severe model of SMA.

Effects of SMN replacement on MN survival. To correlate clinical evolution with histological data, the effect of SMN treatment on the number of MNs in the spinal cord and facial nucleus was evaluated. The number of facial and spinal MNs was therefore compared in lentivector-LacZ-treated and lentivector-SMN-treated SMA mice at a time when they reached the fatal clinical end point (Figure 4). In healthy WT littermates, an average of 55 choline acetyltransferase-positive (ChAT-positive) MNs were present per facial nucleus section (Figure 4F). Cell counts at the end stage of disease revealed that ChAT-positive MN numbers per brain stem section were significantly higher in lentivector-SMN-treated than in lentivector-LacZ control mice, with a notable $120 \%$ increase in MN survival $(38 \pm 2.5$ versus $17.2 \pm 4.2$ ChAT-positive MNs; $n=4$; $P<0.001$; Figure 4, A, B, and F). The lumbar MNs are normally affected early in the disease course in SMA mice. Thus, as expected, at the preterminal end stage of disease, only minimal - though still statistically significant - differences in the lumbar MN cell counts were detected between lentivector-LacZ-treated and lentivector-SMN-treated animals (Figure 4, C-E).

$S M N$ gene therapy induces a minimal immune response. Lentivector-mediated gene transfer in 2-day-old SMA mice induced a minimal immune response in muscles. Specific Ab markers were used to detect immune responsive cells at the site of injection at the end stage of disease. In no cases after EIAV viral delivery were any adverse muscle or spinal cord pathology observed (Figure 5). Intramuscular injections of lentivector-SMN and production of SMN protein (Figure $3 \mathrm{~B}$ ) caused negligible immune reaction, consisting only of a small infiltration of $\mathrm{FA} 11^{+}$macrophages (Figure $5 \mathrm{~A}$ ) and $\mathrm{CD}^{+} \mathrm{T}$ cells (Figure 5B) around the needle tract. In addition, very few or no P7/7-MHC class II-positive cells were observed in the injected muscles (Figure 5C). breathing problems. Survival analysis in SMA mice injected with lentivector-LacZ or lentivector-SMN revealed that SMN replacement significantly extended the life span by an average of 3 and 5 days compared with lentivector-LacZ-injected and untreated animals, respectively $(18 \pm 1.5$ days versus $15 \pm 0.8$ days and $13 \pm 1.2$ days, respectively; $P<0.05$; Figure $3 \mathrm{~B}$ ).

\section{Discussion}

Our data demonstrate, using anatomical and pathological outcomes, that retrogradely transported lentivector efficiently transduces up to $70 \%$ of all MNs in the lumbar spinal cord and brain stem in the SMA model. When using such a lentivector system, SMN gene transfer achieves substantial restoration of SMN 

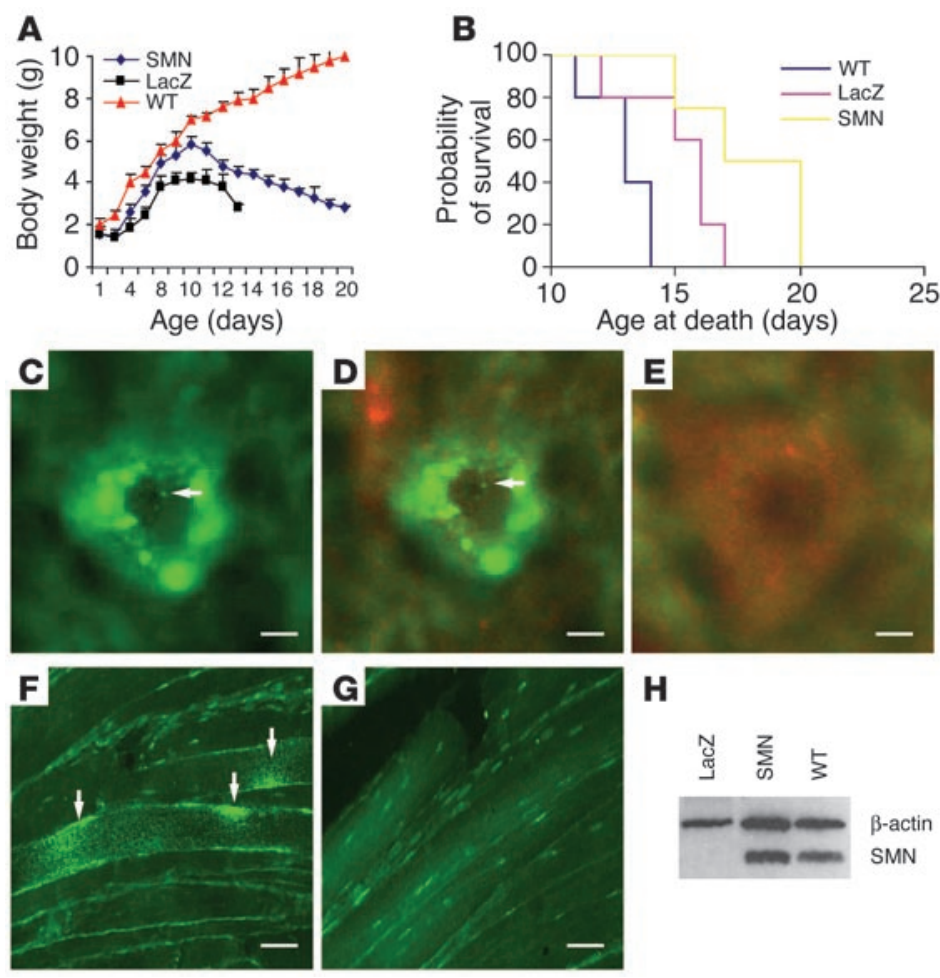

$\mathbf{H}$

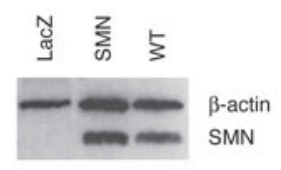

\section{Figure 3}

Retrograde lentivector delivery of SMN at the onset of disease extends survival and delays the phenotype in SMA mice. (A) Weight measurements of animals treated with lentivector-SMN or lentivector-LacZ. (B) Survival analysis in SMA mice injected at 2 days of age with lentivector-LacZ or lentivector-SMN. Mortality was significantly delayed in mice treated with lentivectorSMN compared with the control LacZ group. (C) Image showing lentivector-mediated expression of SMN in spinal MN at the end stage of disease as monitored using Abs against the HA tag. Arrow indicates gems. (D) Double labeling using HA (green, arrow indicates gems) and CGRP Abs (red) in spinal sections. No HA staining was detected in LacZ-injected mice (E). (F and G) SMN expression in muscles from SMN and LacZ-treated mice, respectively. Arrows in $\mathbf{F}$ indicate lentivector-mediated SMN expression. (H) Western blot analysis of ventral spinal cord in LacZ, SMN, and WT animals. Scale bars: $50 \mu \mathrm{m}$ (C-E), $100 \mu \mathrm{m}(\mathbf{F}$ and $\mathbf{G})$. expression in the fibroblast cell line from type I SMA patients. We report here, we believe for the first time, that these vectors can thus be successfully used for SMN gene therapy in a SMA mouse model. Lentivector expressing SMN induces extension of survival in a clinically relevant model of type I SMA. In particular, SMN treatment close to onset of disease delayed onset of disease and increased life expectancy of SMA mice.

Despite worldwide efforts, SMA is, to date, still incurable, in part because of its severity and because the methods to deliver therapeutically attractive molecules are still inefficient. So far, only 1 gene therapy study has been reported in the field (19). In a previous study (19), gene transfer of cardiotrophin-1, using intramuscular injection of adenoviral vectors, induced extension of the life span of another mouse SMA model (20) 30\% (19). Theoretically, the $S M N$ replacement approach is straightforward and easy to achieve. This approach represents a medical challenge, however, when considering that SMA mice develop a phenotype 5 days after birth, first by a decrease in their body weight, followed by MN loss starting at 9 days of age. As the disease progresses, they also develop proximal muscle weakness and atrophy, resulting in end-stage paralysis and death at approximately 13.27 days of age. To explain the minimal effect

\section{Figure 4}

SMN gene replacement protects spinal and brain stem MNs in SMA mice. ChAT immunoreactivity in lentivector-SMN-treated (A, insert) and lentivector-Lac-Z-treated (B, insert) mice. Immunohistochemistry showing CGRP-positive MNs in lumbar spinal cord of lentivector-SMN-injected (C) and lentivector-LacZ-injected (D) animals. (E) Cell counts of surviving lumbar spinal cord MNs in WT (control), lentivector-Lac-Z-, and lentivector-SMN-treated SMA mice at end stage of disease. (F) Quantification of facial nucleus MNs in control, lentivector-LacZ-, and lentivectorSMN-injected animals at end stage of disease. Scale bars: $100 \mu \mathrm{m}$. of SMN replacement on survival of the SMA mouse model, we could propose the following possibilities: (a) SMN expression may need to be restored not only in MNs but also in other cell types such as glia (21); (b) the time window in SMA mice is too short for full SMN expression to occur and spare more MNs (in the present study, lentivector-SMN administration was performed at P2; however, an early treatment, for example, in utero, could improve further the survival of these mice); (c) it has been previously reported in an animal model of another MN disease (ALS) that an increased metabolic demands (hypermetabolism) could be a factor in increasing MN vulnerability (22). It appears, therefore, that other factors could contribute to $\mathrm{MN}$ diseases.
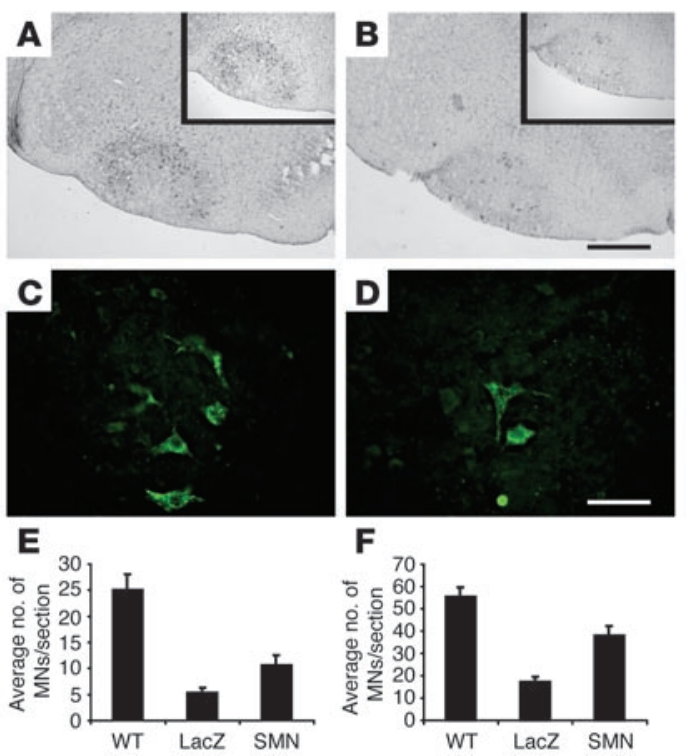

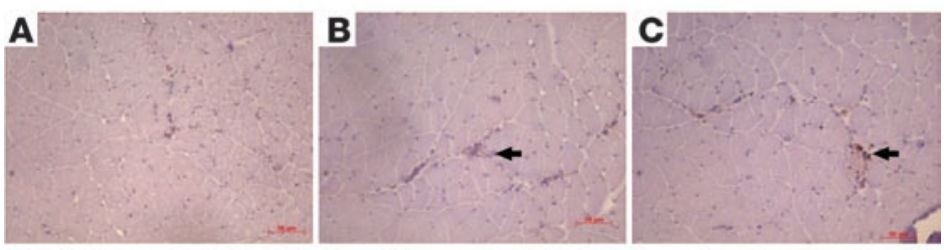

Figure 5

Immune response in SMA mice 1 week after intramuscular lentivector-SMN delivery. Abs used to detect components of the immune response in the injected area of muscle were (A) P7/7: MHC class II; (B) CD3: T cells; and (C) FA11: macrophage. All animals exhibited a minor infiltration at site of injections (arrows). Scale bars: $100 \mu \mathrm{m}$.

Typically, delivery of recombinant growth factors such as GDNF and IGF-I has resulted in marginal therapeutic efficacy in clinical trials for MN diseases such as ALS, presumably because of their short half-life and poor access to MNs (23). The present gene delivery methodology circumvents such needs and problems, and our findings even suggest that targeted delivery of therapeutic candidates in the vicinity of degenerating $\mathrm{MN}$ cell bodies by intramuscular injection of retrogradely transported viral vectors may be helpful and, perhaps, even critical to obtain therapeutic efficiency. In support of this suggestion, we previously demonstrated, using the same methodology, that lentivector-mediated VEGF gene therapy achieves one of the highest therapeutic effects on survival in the ALS mouse model reported to date (12). We believe that this approach may have potential as a safe and practical treatment for many of the motor symptoms of human SMA.

\section{Methods}

Viral production. EIAV self-inactivating vector genomes were constructed from pONY8.0Z or pONY8.0G vectors described previously $(10,24)$. The cDNA coding for the reporter gene LacZ or the human $S M N$ was cloned in the EIAV transfer vector. A EIAV vector genome has been generated that encodes the SMN gene with or without a HA tag. Viral vector stocks pseudotyped with rabies G glycoprotein were prepared using the HEK293T transient system as previously described $(10,25)$. The titers (approximately $4 \times 10^{9}$ T.U./ml) of concentrated EIAV-LacZ viral vectors were estimated by transduction of D17 cells. The titers $\left(4 \times 10^{9}\right.$ to $\left.7 \times 10^{9} \mathrm{~T} . \mathrm{U} . / \mathrm{ml}\right)$ of the EIAV-SMN or EIAV-EIAV-SMN-HA vectors were estimated using real-time quantitative RT-PCR by comparison to EIAV-LacZ vectors and were normalized for viral RNA $(26,27)$.

In vitro transduction. GM03813 fibroblasts from a type 1 SMA patient (Coreill Cell Repository) were transduced with either EIAV-SMN, EIAV-SMN-HA, or EIAV-LacZ vectors pseudotyped with the rabies-G (ERAwt) envelope at an MOI of 20 and 100, respectively, in the presence of $8 \mu \mathrm{g} / \mathrm{ml}$ polybrene (25). At 7 days after transduction, the cells were fixed in $4 \%$ paraformaldehyde. SMN expression was monitored using Abs against SMN and HA tag.

Histology and immunohistochemistry. Animals were perfused transcardially with $0.9 \% \mathrm{NaCl}$ solution followed by ice-cold $4 \%$ paraformaldehyde. Spinal cord, brain, and muscle tissues were dissected out, postfixed overnight in the same solution, and then transferred to $30 \%$ sucrose. Tissues were analyzed by immunohistochemistry and X-gal reaction. Spinal cord and brain tissue were cut on a sliding cryostat microtome (Leica) at a thickness of $20 \mu \mathrm{m}$ and collected as free-floating sections in PBS containing sodium azide. Primary Abs were used as follows: mouse anti-HA (1:100; Roche Diagnostics); mouse anti-SMN (1:1,000; Transduction Laboratories); rabbit anti- $\beta$-gal (1:1,000; Cortex Biochem Inc.); rabbit anti-CGRP (1:3,000; Sigma-Aldrich); mouse anti-ChAT (1:1,000; gift from B.K. Hartman and C. Cozari, University of Minnesota, Minneapolis, Minnesota, USA); CD3-T cells (1:50; Dako Corp.); and P7/7-MHCII (1:100; Dako Corp.).

SMA animal model. All animal procedures were carried out according to United Kingdom Home Office regulations. The SMA mouse model used in the current study was generated by Le and colleagues (13). Briefly, an SMN cDNA lacking exon 7 was placed under the control of a 3.4-kb SMN promoter fragment (transgene referred to as $\mathrm{SMN} \Delta 7$ ) and microinjected into fertilized mouse oocytes (FVB/N). All lines had multiple copies of the transgene as determined by real-time PCR. The SMN $\Delta 7$ transgenic mice were crossed with mice containing SMN2 and a mouse Smn KO allele (8) to obtain doubletransgenic mice $S M N \Delta 7$ SMN2 $S m n^{+/-}$on a FVB/N background backcrossed for 6 generations. These double-transgenic mice were interbred to obtain $\mathrm{Smn}^{-1-}$ mice with SMN2 and SMN $\Delta 7$. Smn ${ }^{-/-} \mathrm{SMN} 2$ mice carrying 2 copies of SMN2 transgene survive an average of 5.16 days after birth (9). SMN $\Delta 7$ was found to extend the survival of Smn ${ }^{-/}$SMN2 mice from $5.16 \pm 0.24$ days to $13.27 \pm 0.34$ days (13).

Intramuscular viral delivery. Rabies $G$ pseudotyped lentiviral vectors carrying human SMN or LacZ gene were injected bilaterally into the hind limb (30 $\mu \mathrm{l}$ each), facial (10 $\mu \mathrm{l})$, diaphragm $(20 \mu \mathrm{l})$, intercostal (10 $\mu \mathrm{l})$, and tongue muscles $(5 \mu \mathrm{l})$ of SMA transgenic mice at P2. Lentivector-SMN or lentivector-LacZ vectors were injected bilaterally into the hind limb gastrocnemius, diaphragm, intercostal, facial, and tongue muscles of SMA mice at disease onset at 2 days of age. Each muscle received single injections into multiple sites. A first group of SMA mice received injections of EIAV$\operatorname{SMN}(n=5)$ at P2. Another group of SMA animals was treated with EIAVLacZ vector $(n=5)$. Untreated SMA mice were also included in the present study $(n=5)$. Injections were done by an operator that was not involved in the further follow-up of animals.

Cell counts. All cell counts were assessed blind by 2 independent observers. In brief, the number of transduced cells were counted in every fifth section throughout the entire lumbar spinal cord and facial nucleus. The counts were performed at a magnification of $\times 40$. The coefficient of error was determined as described previously by Abercrombie (28).

Statistical analysis. Data were expressed as the mean \pm SEM, and statistical analysis was performed using a 1-way ANOVA for comparison between treatment groups. The significance level was set at $P$ values less than 0.05 .

\section{Acknowledgments}

This work was supported by Oxford BioMedica and a grant from Andrews Buddies/FightSMA. NIH grant 38650 supports T. Le and the animal colony used in the current study.

Received for publication August 4, 2004, and accepted in revised form October 18, 2004.

Address correspondence to: Mimoun Azzouz or Nicholas Mazarakis, Oxford BioMedica Ltd., Medawar Centre, Robert Robinson Avenue, The Oxford Science Park, Oxford OX4 4GA, United Kingdom. Phone: 01-865-78-30-00; Fax: 01-865-78-30-01; E-mail:m.azzouz@oxfordbiomedica.co.uk orn.mazarakis@ oxfordbiomedica.co.uk.

Lucy Walmsley's present address is: Oxxon Pharmaccines Ltd., Oxford, United Kingdom.

Umrao R. Monani's present address is: Department of Neurology, Columbia University, College of Physicians and Surgeons, New York, New York, USA. 
1. Gendron, N.H., and MacKenzie, A.E. 1999. Spinal muscular atrophy: molecular pathophysiology. Curr. Opin. Neurol. 12:137-142.

2. Sendtner, M. 2001. Molecular mechanisms in spinal muscular atrophy: models and perspectives. Curr. Opin. Neurol. 14:629-634.

3. Lefebvre, S., et al. 1995. Identification and characterization of a spinal muscular atrophy-determining gene. Cell. 80:155-165.

4. Roberts, D.F., Chavez, J., and Court, S.D. 1970. The genetic component in child mortality. Arch. Dis. Child. 45:33-38.

5. Munsat, T.L., and Davies, K.E. 1992. International SMA consortium meeting. Neuromuscul. Disord. 2:423-428.

6. Lorson, C.L., Hahnen, E., Androphy, E.J., and Wirth, B. 1999. A single nucleotide in the SMN gene regulates splicing and is responsible for spinal muscular atrophy. Proc. Natl. Acad. Sci. U. S. A. 96:6307-6311.

7. Monani, U.R., et al. 1999. A single nucleotide difference that alters splicing patterns distinguishes the SMA gene SMN1 from the copy gene SMN2. Hum. Mol. Genet. 8:1177-1183.

8. Schrank, B., et al. 1997. Inactivation of the survival motor neuron gene, a candidate gene for human spinal muscular atrophy, leads to massive cell death in early mouse embryos. Proc. Natl. Acad. Sci. U. S. A. 94:9920-9925.

9. Monani, U.R., et al. 2000. The human centromeric survival motor neuron gene (SMN2) rescues embryonic lethality in Smn(-/-) mice and results in a mouse with spinal muscular atrophy. Hum. Mol. Genet. 9:333-339.

10. Mazarakis, N.D., et al. 2001. Rabies virus glycoprotein pseudotyping of lentiviral vectors enables retrograde axonal transport and access to the nervous system after peripheral delivery. Hum. Mol. Genet. 10:2109-2121.

11. Wong, L.F., et al. 2004. Transduction patterns of pseudotyped lentiviral vectors in the nervous system. Mol. Ther. 9:101-111.

12. Azzouz, M., et al. 2004. VEGF delivery with retrogradely transported lentivector prolongs survival in a mouse ALS model. Nature. 429:413-417.

13. Le, T., et al. 2005. Expression of SMN $\Delta 7$, the major product of the centromeric survival motor neuron gene (SMN2), extends survival in mice with spinal muscular atrophy. In press.

14. Battaglia, G., Princivalle, A., Forti, F., Lizier, C., and Zeviani, M. 1997. Expression of the SMN gene, the spinal muscular atrophy determining gene, in the mammalian central nervous system. Hum. Mol. Genet. 6:1961-1971.

15. Lefebvre, S., et al. 1997. Correlation between severity and SMN protein level in spinal muscular atrophy. Nat. Genet. 16:265-269.

16. Liu, Q., and Dreyfuss, G. 1996. A novel nuclear structure containing the survival of motor neurons protein. EMBO J. 15:3555-3565.

17. Coovert, D.D., et al. 1997. The survival motor neuron protein in spinal muscular atrophy. Hum. Mol. Genet. 6:1205-1214.

18. Patrizi, A.L., et al. 1999. SMN protein analysis in fibroblast, amniocyte and CVS cultures from spinal muscular atrophy patients and its relevance for diagnosis. Eur. J. Hum. Genet. 7:301-309.

19. Lesbordes, J.C., et al. 2003. Therapeutic benefits of cardiotrophin-1 gene transfer in a mouse model of spinal muscular atrophy. Hum. Mol. Genet. 12:1233-1239.

20. Frugier, T., et al. 2000. Nuclear targeting defect of
SMN lacking the C-terminus in a mouse model of spinal muscular atrophy. Hum. Mol. Genet. 9:849-858

21. Clement, A.M., et al. 2003. Wild-type nonneuronal cells extend survival of SOD1 mutant motor neurons in ALS mice. Science. 302:113-117.

22. Dupuis, L., et al. 2004. Evidence for defective energy homeostasis in amyotrophic lateral sclerosis: benefit of a high-energy diet in a transgenic mouse model. Proc. Natl. Acad. Sci. U. S. A. 101:11159-11164.

23. Mitchell, J.D., Wokke, J.H., and Borasio, G.D. 2002. Recombinant human insulin-like growth factor I (rhIGF-I) for amyotrophic lateral sclerosis/motor neuron disease [review]. Cochrane Database Syst. Rev. 3:CD002064

24. Azzouz, M., et al. 2002. Multicistronic lentiviral vector-mediated striatal gene transfer of aromatic L-amino acid decarboxylase, tyrosine hydroxylase, and GTP cyclohydrolase I induces sustained transgene expression, dopamine production, and functional improvement in a rat model of Parkinson's disease. J. Neurosci. 22:10302-10312.

25. Mitrophanous, K., et al. 1999. Stable gene transfer to the nervous system using a non-primate lentiviral vector. Gene Ther. 6:1808-1818.

26. Rohll, J.B., et al. 2002. Design, production, safety, evaluation, and clinical applications of nonprimate lentiviral vectors. Methods Enzymol. 346:466-500.

27. Martin-Rendon, E., Azzouz, M., and Mazarakis, N.D. 2001. Lentiviral vectors for the treatment of neurodegenerative diseases. Curr. Opin. Mol. Ther. 3:476-481.

28. Abercrombie, M. 1948. Estimation of nuclear population from microtome sections. Anat. Rec. 94:239-247. 